Full length article

\title{
Laminin mimetic peptide nanofibers regenerate acute muscle defect
}

\author{
Cagla Eren Cimenci ${ }^{\mathrm{a}, \mathrm{b}}$, Gozde Uzunalli ${ }^{\mathrm{a}, \mathrm{b}}$, Ozge Uysal ${ }^{\mathrm{a}, \mathrm{c}}$, Fatih Yergoz ${ }^{\mathrm{a}, \mathrm{b}}$, Ebru Karaca Umay ${ }^{\mathrm{d}}$, \\ Mustafa O. Guler ${ }^{\mathrm{e}, *}$, Ayse B. Tekinay ${ }^{\mathrm{a}, \mathrm{b}, *}$
}

a Institute of Materials Science and Nanotechnology, National Nanotechnology Research Center (UNAM), Bilkent University, Ankara 06800, Turkey

${ }^{\mathrm{b}}$ Materials Science and Nanotechnology Graduate Program, Bilkent University, Ankara 06800, Turkey

${ }^{\mathrm{c}}$ Neuroscience Graduate Program, Bilkent University, Ankara 06800, Turkey

${ }^{\mathrm{d}}$ Diskapi Yildirim Beyazit Training and Research Hospital, Physical Medicine and Rehabilitation Clinic, Ankara 06800, Turkey

e Institute for Molecular Engineering, University of Chicago, Chicago, IL 60637, USA

\section{A R T I C L E I N F O}

\section{Article history:}

Received 5 February 2017

Received in revised form 30 June 2017

Accepted 5 July 2017

Available online 8 July 2017

\section{Keywords:}

Peptide nanofiber

Acute muscle injury

Regeneration

Laminin

Hydrogel

Functional self-assembly

\begin{abstract}
A B S T R A C T
Skeletal muscle cells are terminally differentiated and require the activation of muscle progenitor (satellite) cells for their regeneration. There is a clinical need for faster and more efficient treatment methods for acute muscle injuries, and the stimulation of satellite cell proliferation is promising in this context. In this study, we designed and synthesized a laminin-mimetic bioactive peptide (LM/E-PA) system that is capable of accelerating satellite cell activation by emulating the structure and function of laminin, a major protein of the basal membrane of the skeletal muscle. The LM/E-PA nanofibers enhance myogenic differentiation in vitro and the clinical relevance of the laminin-mimetic bioactive scaffold system was demonstrated further by assessing its effect on the regeneration of acute muscle injury in a rat model. Laminin mimetic peptide nanofibers significantly promoted satellite cell activation in skeletal muscle and accelerated myofibrillar regeneration following acute muscle injury. In addition, the LM/E-PA scaffold treatment significantly reduced the time required for the structural and functional repair of skeletal muscle. This study represents one of the first examples of molecular- and tissue-level regeneration of skeletal muscle facilitated by bioactive peptide nanofibers following acute muscle injury.
\end{abstract}

\section{Significance Statement}

Sports, heavy lifting and other strength-intensive tasks are ubiquitous in modern life and likely to cause acute skeletal muscle injury. Speeding up regeneration of skeletal muscle injuries would not only shorten the duration of recovery for the patient, but also support the general health and functionality of the repaired muscle tissue. In this work, we designed and synthesized a laminin-mimetic nanosystem to enhance muscle regeneration. We tested its activity in a rat tibialis anterior muscle by injecting the bioactive nanosystem. The evaluation of the regeneration and differentiation capacity of skeletal muscle suggested that the laminin-mimetic nanosystem enhances skeletal muscle regeneration and provides a suitable platform that is highly promising for the regeneration of acute muscle injuries. This work demonstrates for the first time that laminin-mimetic self-assembled peptide nanosystems facilitate myogenic differentiation in vivo without the need for additional treatment.

() 2017 Acta Materialia Inc. Published by Elsevier Ltd. All rights reserved.

\section{Introduction}

Skeletal muscle is one of the most abundant types of tissues in the human body and facilitates a majority of voluntary move-

\footnotetext{
* Corresponding authors at: Institute of Materials Science and Nanotechnology, National Nanotechnology Research Center (UNAM), Bilkent University, Ankara 06800, Turkey (A.B. Tekinay), Institute for Molecular Engineering, University of Chicago, Chicago, IL 60637, USA (M.O. Guler).

E-mail addresses: mguler@uchicago.edu (M.O. Guler), atekinay@bilkent.edu.tr (A.B. Tekinay)
}

ments. It has a hierarchical fiber organization that contains multi-nucleated and parallel-aligned muscle fibers in each muscle. Satellite cells are skeletal muscle progenitor cells that are found between the basal lamina and the plasma membrane, and promote the growth, repair and regeneration of skeletal muscle fibers [1,2]. They are unipotent cells and exhibit a strong capacity for selfrenewal, which allows them to maintain a stable population while re-establishing muscle fiber structure following injury. Satellite cells are activated following injury to skeletal muscle and play important roles in the degeneration, inflammation, regeneration 
and remodeling steps that constitute the muscle repair process [3]. Muscle fibers are regenerated through the fusion of satellite cells with existing fibers, producing multinucleated, post-mitotic and highly differentiated muscle tissue. This fusion is triggered by the temporally ordered changes in the gene expression profiles of myoblast cells that are responsible for the formation of myotubes $[4,5]$. In addition to being adapted to maintain contractile activity in a variety of environmental conditions, skeletal muscle exhibits a spectacular regeneration capacity in response to small injuries. In such cases, restoration of the slightly damaged muscle is possible without any therapeutic intervention [6,7]. However; if damage is severe and left untreated, it may result in the loss of muscle mass and function. In these cases, skeletal muscle regeneration cannot be completed without the help of medical treatment or surgical reconstruction [8]. Accordingly, the development of effective treatment methods against major skeletal muscle injuries is a topic of considerable interest.

Developments in biomaterials technology have made it possible to fabricate materials that can efficiently facilitate the regeneration of various tissue types [1]. These scaffolds can be designed to exhibit a broad range of physiochemical and biological characteristics, depending on the tissue they are intended to support. As such, both natural and synthetic biomaterials have been used to mimic the architectural and biomechanical features of various tissues of the human body $[9,10]$.

The skeletal muscle extracellular matrix (ECM) is composed of a variety of proteoglycans and proteins. Laminin is a major ECM element in skeletal muscle tissue and binds directly to collagen IV [11]. Laminins constitute a group of glycoproteins that are found in abundance in all basement membranes, and skeletal muscle laminins serve to protect muscle fibers from external damage and facilitate their regeneration following injury [12]. Laminin was previously shown to have a crucial role in myogenesis by triggering the fusion of satellite cells, resulting in proliferation and skeletal muscle repair $[13,14]$. In some skeletal muscle studies, fibronectin has also been reported to regulate satellite cell expansion [15]. However, we had previously observed that laminin epitope "IKVAV" is able to stimulate the expansion of satellite cells to a greater extent than fibronectin epitope "RGD" [16].

The IKVAV peptide sequence is derived from $\beta 1$ and $\alpha 1$ cell binding domains of laminin and has been utilized to mimic the laminin function of skeletal muscle tissue [17]. The natural ECM consists of complex signals that interact with each other to organize cellular behavior and responses. Considering their biocompatibility, adaptability and general similarity to structural proteins, peptide nanofibers are considered to be ideal materials for the design of artificial extracellular matrix-like scaffolds [18,19]. It is possible to design a complex nanonetwork system through the programmed self-assembly of peptide amphiphile molecules, which is driven by several noncovalent interactions [20]. Under physiological conditions, higher-order complex nanostructures are formed from primary amino acid sequences and effectively replicate the biochemical characteristics and architecture of native tissues. Owing to their special characteristics, peptide amphiphile molecules are considered to be excellent bioactive scaffold candidates for tissue engineering and regenerative medicine applications [21,22]. Peptide sequences derived from the active site of the laminin protein have been reported to exhibit crucial roles in in vitro muscle regeneration, possibly by mimicking the environment and bioactive signals provided by the basal lamina of skeletal muscle. It was also shown that laminin-mimetic PA was not toxic and favored the adhesion, growth and differentiation of myoblasts [16]. In addition, the material did not inhibit the differentiation of satellite cells. As such, the peptide material in question (LM/E-PA) was selected as a potential candidate for in vivo regeneration of damaged skeletal muscle. In this work, we utilized laminin- mimetic peptide nanofiber scaffold system to enhance the regeneration of acute muscle injury. The regeneration and differentiation capacity of skeletal muscle was evaluated by behavioral, histological/physiological and molecular analyses. This work demonstrates for the first time that injection of laminin-mimetic self-assembled peptide nanofiber network facilitates myogenic differentiation and muscle regeneration in vivo without necessitating additional treatment.

\section{Materials \& methods}

9-Fluorenylmethoxycarbonyl (Fmoc) and tert-butoxycarbonyl (Boc) protected amino acids, lauric acid, [4-[ $\alpha-\left(2^{\prime} 4^{\prime}\right.$-dimethoxyphe nyl) Fmoc-amino methyl] phenoxyl] acetomidonorleucyl-MBHA resin (Rink amide MBHA resin), Fmoc-Asp (OtBu)-Wang resin and 2-(1H-benzotriazol-1-yl)-1,1,3,3-tetramethyluronium hexafluorophosphate (HBTU) and diisopropylethylamine (DIEA) were purchased from Merck, NovaBiochem, Sigma-Aldrich and ABCR. Cover glasses and tissue culture plates were purchased from NEST Biotechnology and Corning. Antibodies were obtained from Abcam. Chondrogenesis Differentiation Kit was purchased from Thermo Fisher Scientific. All other materials and chemicals used in the study were analytical grade and purchased from Invitrogen, Fisher, Merck and/or Sigma-Aldrich. All the chemicals were used as provided.

\subsection{Peptide amphiphile synthesis, purification and characterization}

Peptide amphiphile molecules were synthesized manually by standard solid phase peptide synthesis chemistry [23]. Laminin mimetic PA (LM-PA, Lauryl-VVAGKKIKVAV-Am) was constructed on Rink amide MBHA resin and E-PA (Lauryl-VVAGE) was constructed on Fmoc-Asp(OtBu)-Wang resin. Couplings of amino acids were performed with 2 equivalents of amino acids activated with 1.95 equivalents of HBTU, and 3 equivalents of $\mathrm{N}, \mathrm{N}$ diisopropylethylamine (DIEA) for one unit of starting resin. Each amino acid coupling proceeded for $2 \mathrm{~h}$ and cleavage of Fmoc protecting group was performed by treating the solid phase with $20 \%(\mathrm{v} / \mathrm{v})$ piperidine in dimethylformamide (DMF) for $20 \mathrm{~min}$. After coupling all Fmoc protected amino acids, alkyl tail was attached by using lauric acid addition similarly to amino acid coupling except that coupling time was $4 \mathrm{~h}$. In order to block remaining free amine groups by acetylating the unreacted amine groups after each amino acid coupling, $10 \%(\mathrm{v} / \mathrm{v})$ acetic anhydride solution in DMF was added and treated for $30 \mathrm{~min}$. After each step, resin was washed with DMF, DCM and DMF respectively (three times each). Cleavage of protecting groups and peptide molecules from the resin was carried out by $95 \%$ trifluoroacetic acid (TFA) containing cleavage cocktail (95\% TFA, 2.5\% triisopropylsilane (TIS), $2.5 \%$ water) for $3 \mathrm{~h}$. Excess TFA was removed by rotary evaporation. As a following step, ice-cold diethyl ether was used to precipitate the remaining viscous PA solution overnight at $-20^{\circ} \mathrm{C}$. Diethyl ether was expelled after centrifugation at $8000 \mathrm{rpm}$ for $15 \mathrm{~min}$ and the resulting precipitate was dissolved in $\mathrm{dd}_{2} \mathrm{O}$ and freezedried for two days.

Lyophilized peptide amphiphile material was characterized by liquid chromatography-mass spectrometry (LC-MS) and mass spectrum was obtained with Agilent 6530 quadrupole time of flight (Q-TOF) mass spectrometry with electrospray ionization (ESI) source equipped with reverse-phase HPLC system with Zorbax Extend-C18 $21.2 \times 150 \mathrm{~mm}$ column for basic conditions and Zorbax SB-C8 $21.2 \times 150 \mathrm{~mm}$ column for acidic conditions. To purify the PA molecules and remove the residual TFA, preparative HPLC system (Agilent 1200 series) was used with a mobile phase of optimized gradient $0.1 \% \mathrm{TFA} /$ water and $0.1 \% \mathrm{TFA} /$ acetonitrile 
for acidic conditions or $0.1 \%$ ammonium hydroxide/water and $0.1 \%$ ammonium hydroxide/acetonitrile for basic conditions. $0.1 \% \mathrm{HCl}$ treatment was processed for positively charged peptide amphiphiles. After purification step, PAs were lyophilized and stored in a powder form at $-20^{\circ} \mathrm{C}$ for further use.

\subsection{Self-assembled nanofiber network formation}

Peptide amphiphile solutions were prepared by dissolving PAs in sterile $\mathrm{ddH}_{2} \mathrm{O}$ and sonicated for $15 \mathrm{~min}$. In order to form neutrally charged peptide nanofibers, positively charged bioactive LM-PA and negatively charged E-PA (at pH 7.4) were mixed to form nanofibers at 2:3 $\mathrm{M}$ ratio, respectively. Mixing process was carried out within the syringe right before injection.

\subsection{Structural and mechanical characteristics of peptide nanofibers}

PA nanofiber network was observed by imaging with a scanning electron microscope (SEM). To prepare the sample, oppositely charged PA solutions (1 wt\%) were mixed on the silicon wafer surface to produce gel with neutral charge as explained above. After waiting for $10 \mathrm{~min}$ of gelation, hydrogel was dehydrated in graded ethanol solutions $(20 \%, 40 \%, 60 \%, 80 \%$ and $100 \% \mathrm{v} / \mathrm{v})$ for $10 \mathrm{~min}$ at each step. After dehydration, gel was critical point dried by using Autosamdri ${ }^{\circledR}-815 B$ from Tousimis. Dried gel was coated with $6 \mathrm{~nm} \mathrm{Au} / \mathrm{Pd}$ and SEM (FEI Quanta 200 FEG) images were taken by using an Everhart-Thornley Detector (ETD) at high vacuum mode.

For transmission electron microscopy (TEM) analysis, $1 \mathrm{mM}$ LM-PA and $1 \mathrm{mM}$ E-PA were mixed at 2:3 ratio. Sample was put on a 200 -mesh carbon TEM grid for $1 \mathrm{~min}$ followed by $2 \mathrm{wt} \%$ uranyl acetate staining for $40 \mathrm{~s}$ and drying under flow hood. Nanofiber was imaged with TEM (FEI Tecnai G2 F30 TEM).

In order to probe the viscoelastic properties of the PA network, oscillatory rheology measurement was performed with an Anton Paar Physica RM301 rheometer operating with a $25 \mathrm{~mm}$ parallel plate configuration at $25^{\circ} \mathrm{C}$. Samples of LM-PA and E-PA were mixed within the syringe at $2: 3 \mathrm{M}$ ratio and placed on the lower stage of the rheometer. Measurement was done with $0.5 \mathrm{~mm}$ gap distance, $100-0.1 \mathrm{rad} / \mathrm{s}$ angular frequency and $0.5 \%$ shear strain. Storage moduli $\left(G^{\prime}\right)$ and loss moduli $\left(G^{\prime \prime}\right)$ values were scanned and recorded at each strain.

Circular dichroism (CD) (JASCO J815 CD spectrapolarimetry) was used to analyze the secondary structures of PA molecules. To perform the analysis, $0.2 \mathrm{mM}$ aqueous solutions of peptide amphiphiles were diluted from $1 \mathrm{mM}$ stock solutions. For each measurement, $300 \mu \mathrm{L}$ of the sample was put into a $1 \mathrm{~mm}$ quartz cuvette. Scanning was done between $190 \mathrm{~nm}$ to $300 \mathrm{~nm}$, data interval and data pitch were $0.1 \mathrm{~nm}$ and all measurements were performed with three accumulations. Molar ellipticity was calculated with the data obtained from measurements using the equation: $[\theta]$ $=100 \times \theta /(C \times l)$, where $C$ is the concentration in molar, and $l$ is the cell path length in centimeters.

\subsection{In vivo acute muscle injury model}

Animal studies were performed in the center of Diskapi Yildirim Beyazit Research and Training Hospital for animal care and handling (ADACELL), with protocols approved by local and governmental Ethics Committees. 50 male, 2 months old and healthy Sprague-Dawley rats were used in this study $(n=10)$, weighing 200-250 g. Male rats were chosen to eliminate the potential contradictory effect of hormonal fluctuations on the body in this age range. Rats were housed in standard plastic cages at a temperature and light controlled environment $\left(24^{\circ} \mathrm{C} ; 12 / 12 \mathrm{~h}\right.$ light/dark cycle) and they were allowed to access food and water ad libitum throughout the experiment.

\subsection{Toxin injection}

Following the anesthesia with ketamine $(100 \mathrm{mg} / \mathrm{kg})$ and xylazine $(10 \mathrm{mg} / \mathrm{kg})$, the anterolateral side of the hindlimbs were shaved and treated with an aseptic solution. A longitudinal incision was made to expose the tibialis anterior (TA) muscle (Supp. Fig. 1A). TA muscle was injured by the injection of $150 \mu \mathrm{L}$ $(10 \mu \mathrm{M})$ cardiotoxin (from Naja Mosambica Mosambica). After acute muscle injury formation, the skin was closed with a 4-0 suture. Rats were then randomly divided into 5 groups: Day $1,3,5,7$ and 14 .

\subsection{Treatment with ECM mimetic PA network}

A day after the operation, right legs of the each animal were treated with the $150 \mu \mathrm{L}$ laminin mimetic PA hydrogel application. Gel was made by mixing LM-PA and E-PA into the syringe. Likewise, left legs of the animals were used as a negative control group and $150 \mu \mathrm{L}$ of physiological saline was applied to ensure that damage and recovery conditions were as similar as possible in both groups. Non-bioactive peptide was not tested as a control in this study, as it has been shown not to promote myogenesis in our previous work [16]. In order to inject the hydrogel and physiological saline to the correct location, a longitudinal incision was made to the damaged area. After treatment, the skin was closed with a 40 suture. All animals were carefully evaluated during the first $24 \mathrm{~h}$ after operation. No animals were observed to be infected throughout the experimental period.

\subsection{Electromyography (EMG) analysis}

Electrophysiological studies were performed to measure the amplitude of the compound motor action potentials (CMAP). Measurements were taken by using Neuro-MEP-Micro two channels EMG (Neurosoft, Russia) device. Recordings were collected at periods of pre-operation (healthy), right after the operation, day 7 and day 14 post-operation. After anesthesia with ketamine $(100 \mathrm{mg} /$ $\mathrm{kg})$ and xylazine $(10 \mathrm{mg} / \mathrm{kg})$, evaluation site was cleaned with alcohol and allowed to dry before procedure. For this evaluation; monopolar needle recording electrode was placed in tibialis anterior muscle, reference electrode was placed in dorsum of the foot and common reference (ground electrode) was placed on the tail. In order to observe the amplitude of the motor action potentials, sweep speed and sensitivity were set to $4 \mathrm{mV}$ and $4 \mathrm{~ms} /$ division. The common peroneal nerve found in the knee at fibula's head was stimulated with bipolar concentric needle electrode. The intensity was gradually increased until dorsiflexion movement was observed and up to supramaximal intensity (current intensity of $30 \%$ above the value to evoke the maximal CMAP). CMAP amplitude (negative peak's amplitude) was recorded from the TA muscle. Stimulation was repeated 3 times for all measurements and average values were used for statistical analysis.

\subsection{Walking track step test analysis}

At 1, 3, 5, 7 and 14 days after treatment, animals underwent a postoperative behavioral walking-track analysis. The rats were walked over a white sheet of paper $(8.2 \mathrm{~cm}$ wide, $58 \mathrm{~cm}$ long) covering the bottom of a wooden alley ending in a dark destination box. Hindlimbs of the animals were painted with black ink, and then they were placed into the track to walk. Stride lengths were measured manually as the distance between two pawprints. By considering three longest steps from each walk, stride lengths were calculated [24]. Obtained data were analyzed and processed in Graphpad Prism 5, as the mean with standard deviation for each 
limb. Statistical comparisons were made by paired $t$-test for paired values and unpaired $t$-test for unpaired values.

\subsection{Histological analysis (HEE)}

After 1, 3, 5, 7 and 14 day recovery, 10 rats for each day point were sacrificed and their TA muscles were extracted (Supp. Fig. 1B). TA muscles were divided into 3 pieces in order to proceed with histological, gene expression and protein detection analyses. The sections allocated for histological examination were put into $4 \%$ paraformaldehyde solution for fixation. After $24 \mathrm{~h}$, samples were dehydrated with a graded series of ethanol from $70 \%$ to $100 \%$ and prepared for paraffin embedding with two changes of xylene. Cleared samples were embedded in paraffin blocks and sectioned at $5 \mu \mathrm{m}$ thickness by microtome. To assess regeneration, hematoxylin and eosin staining was performed and slides were mounted with Histomount mounting medium. Cross-sections were prepared for histological examination on Axio Scope (Zeiss) light microscope.

\subsection{Immunohistochemistry}

Before the immunohistological analysis, tissue sections were deparaffinized with xylene and rehydrated through ethanol exchange. Antigen retrieval was performed on the sections with EDTA buffer $(\mathrm{pH}=8)$ in the stream chamber for $30 \mathrm{~min}$. Endogenous peroxidase activity was blocked with $0.3 \%$ hydrogen peroxide for $20 \mathrm{~min}$ at room temperature. Before the blocking step, sections were washed with $0.025 \%$ Triton X-100 in TBS. Sections were then blocked with $10 \%$ Normal Goat Serum (NGS) in $1 \%$ BSA in TBS for $1 \mathrm{~h}$ at room temperature followed by incubation with mouse anti-MHC (1:50, MAB4470, R\&D Systems) antibody overnight at $4{ }^{\circ} \mathrm{C}$. Sections were then washed with $0.025 \%$ Triton X-100 in TBS and incubated with a secondary antibody (HRP conjugated antimouse IgG, 1:500, 12-349, Millipore) for $1 \mathrm{~h}$ at room temperature. Both primary and secondary antibodies are prepared with $1 \%$ BSA in TBS. Sections were washed with $0.025 \%$ Triton X-100 in TBS and incubated with DAB solution for $20 \mathrm{~min}$. Sections were then counterstained in hematoxylin and dehydrated.

\subsection{Muscle cross section area calculation}

We next determined the loss of skeletal muscle mass in response to acute injury. Measurements were conducted at $20 \times$ magnification. Per each cross section, five random fields were chosen and area of fibers were calculated by using Image J software. Changes in muscle fiber cross section area were determined using parametric (2-way ANOVA) test.

\subsection{Centrally located nuclei calculation}

Centrally located myonuclei measurements were conducted on day 7 at $40 \times$ magnification per each cross section by bright-field microscopy. The number of central nuclei per muscle fiber was determined by counting a minimum of five random muscle fiber images per animal and five animals from each group were analyzed. \% of myofibers with central nuclei was calculated and statistical analysis was done by using parametric $t$-test.

\subsection{Gene expression analysis by $q R T-P C R$}

For analyzing myogenic differentiation, gene expression profiles were assessed by quantitative real time PCR (qRT-PCR). Tissue part allocated for gene expression analysis was put into a test tube right after animals were sacrificed and immediately frozen with liquid nitrogen. RNA from each sample was isolated with TRIzol reagent
(Invitrogen) according to the manufacturer's instructions. Purity and yield of isolated RNA were determined with Nanodrop 2000 spectrometer (Thermo Scientific). Both cDNA synthesis and qRTPCR were performed with one-step qRT-PCR kit (SuperScript III Platinum SYBR Green) according to the manufacturer's instructions. Reaction conditions were briefly as follows: $55^{\circ} \mathrm{C}$ for $5 \mathrm{~min}, 95^{\circ} \mathrm{C}$ for $5 \mathrm{~min}, 40$ cycles of $95^{\circ} \mathrm{C}$ for $15 \mathrm{~s}, 60^{\circ} \mathrm{C}$ for $30 \mathrm{~s}$ and $40{ }^{\circ} \mathrm{C}$ for $1 \mathrm{~min}$. Product specificity was confirmed by following a melting curve and the reaction efficiencies for each primer set were evaluated with standard curve using 5-fold serial dilutions of total RNA. For the analysis of expression, primary gene expression data were normalized by the expression level of healthy group and the expression level of GAPDH. A comparative Ct method was used to analyze the results.

\subsection{Protein detection by western blotting}

For detecting myogenic differentiation related proteins, protein profiles were assessed by western blot. Tissue part allocated for protein detection was put into a test tube right after animals were sacrificed and immediately frozen with liquid nitrogen. Total protein from each sample was homogenized with RIPA buffer containing $10 \mathrm{mM}$ Tris- $\mathrm{HCl}$ (pH:8.0), 1 mM EDTA, $0.5 \mathrm{mM}$ EGTA, 0.1\% SDS, $140 \mathrm{mM} \mathrm{NaCl}, 1 \%$ Triton X-100 and $0.1 \%$ sodium deoxycholate supplemented with protease inhibitor cocktail (Sigma). Protein concentrations were determined with BCA Assay (Thermo Scientific) and were diluted so as to reach $50 \mu \mathrm{g} / \mathrm{mL}$ final concentration. Proteins were denaturated, resolved on $12 \%$ SDS-PAGE and transferred to polyvinylidene difluoride membranes (Thermo Scientific). Membranes were blocked with $5 \%$ freeze-dried nonfat milk in TBS-T for $1 \mathrm{~h}$ at room temperature and then incubated with primary antibody at $4^{\circ} \mathrm{C}$ overnight. Anti-Pax7 was purchased from LifeSpan BioSciences (1:5000), anti-MyoD1 was purchased from Abcam $(1 \mu \mathrm{g} / \mathrm{mL})$, anti-Myf5 was purchased from abcam $(1: 10,000)$, Anti-Myogenin was purchased from abcam (1:250) and AntiGAPDH was purchased from Millipore (1:750). Afterwards, membranes were washed with TBS-T extensively and incubated with HRP conjugated secondary antibody for $1 \mathrm{~h}$ and visualized by enhanced chemiluminescence (Bio-Rad) according to the manufacturer's protocol on ChemiDoc ${ }^{\mathrm{TM}}$ Imaging System with Image Lab ${ }^{\mathrm{TM}}$ Software - Bio-Rad. Anti-rabbit IgG-HRP conjugated secondary antibody was purchased from abcam (1:2000) and Anti-mouse IgG-HRP conjugated secondary antibody was purchased from Millipore $(1: 1000)$.

\subsection{Statistical analysis}

Results were expressed as mean \pm standard deviation. Either one-way analysis of variance (ANOVA) or two-way ANOVA with Tukey's/Bonferroni's Multiple Comparison Test (GraphPad Prism v5) was used to compare the values between the study groups. Paired and non-paired $t$-test were also applied to describe the association between the study groups. A value of $\mathrm{p} \leq 0.05$ was considered to be statistically significant.

\section{Results}

3.1. Synthesis of PA molecules and characterization of their selfassembly into nanofibers

Laminin-mimetic PA (LM-PA) and E-PA were designed (Supp. Fig. 2) to replicate the microenvironment of the muscle basal lamina. E-PA is a non-bioactive, epitope-free PA molecule that contains a hydrophobic lauryl tail and a $\beta$-sheet forming peptide sequence (VVAG). Two oppositely-charged peptide molecules form hydro- 
gels through electrostatic interactions at physiological pH [25], and the E-PA was used for hydrogel formation with oppositely charged PAs to obtain LM/E-PA nanofibers. The LM-PA molecule was decorated with the laminin-derived sequence "IKVAV" to generate a myogenic nanofibrous environment suitable for the myogenic differentiation of satellite cells in an in vivo muscle regeneration model. All PAs were synthesized with solid phase peptide synthesis method, purified with HPLC and characterized by LC/MS (Supp. Fig. 3). At pH 7.4, LM-PA has a net theoretical charge of +3 , whereas E-PA bears a -2 net charge. Self-assembly was triggered by mixing $1 \mathrm{wt} \%$ of negatively charged and positively-charged peptides at $\mathrm{pH}$ 7.4 at a ratio of 2:3 (LM-PA:E-PA) to produce neutrally charged peptide networks. Peptide molecules were mixed inside a surgical syringe to produce a ready-to-administer peptide nanofiber for in vivo applications.

\subsection{Structural and mechanical characteristics of peptide nanofibers}

Following their preparation, peptide nanofiber networks were characterized to determine their capacity to mimic the structure and function of the natural ECM. Scanning electron microscopy (SEM) and transmission electron microscopy (TEM) were used to visualize the complex nanofiber network and individual fiber structure of nanotubes (Fig. 1A and B). After self-assembly, both organized structures and individual nanofibers were observed by SEM and TEM. Circular dichroism (CD) analysis was used (Fig. 1C) to characterize the secondary structures of peptide amphiphiles and their mixtures. LM-PA and LM/E-PA showed a $\beta$-sheet structure with a minimum around $220 \mathrm{~nm}$ and maximum around $200 \mathrm{~nm}$. Oscillatory rheology was employed in order to characterize the mechanical properties of the gel (Fig. 1D). We also checked whether mechanical properties were altered by the sam- ple preparation method by employing another method of mixture, in which the peptide solutions were mixed outside the syringe (Supp. Fig. 4) where a stronger form of gel was observed.

\subsection{Animal behavior analyses}

Acute muscle injuries were created in both legs and treated with either LM/E-PA peptide nanofiber injection (right leg) or the injection of physiological saline (left leg) as a negative control. The recovery process was evaluated by step test on days $1,3,5$, 7 and 14 (Fig. 2A and B). During the first 5 days of the experiment, step lengths of both legs were observed to be nearly identical for each time point. Strikingly, the peptide nanofiber treated group showed significantly enhanced step length compared to the saline-treated group at both day 7 and day 14 . Functional recovery rate was 1.2 times higher in the LM/E-PA treated group than salinetreated group, suggesting that the bioactive peptide nanofiber treatment accelerated the rate of muscle repair. Muscle recovery was also quantified by electromyographical analysis at four time points; pre-operation (healthy), right after the operation (day zero), day 7 and day 14 post-operation (Fig. 2C). The healthy group gave the highest amplitude values and no significant differences were observed between the right and left legs. After cardiotoxin (CTX) injection, amplitudes for both right and left legs drastically decreased and no significant difference was observed between the two legs. The minimum amplitude values were also measured in this post-operation group. Notably, the peptide nanofiber group showed significantly higher amplitude values after 7 and 14 days of treatment compared to the saline control. LM/E-PA treated group induced greater functional recovery (96\%) compared with the saline-treated group (69\%). In addition, the amplitude recorded at day 7 for the peptide nanofiber group was comparable to that
A

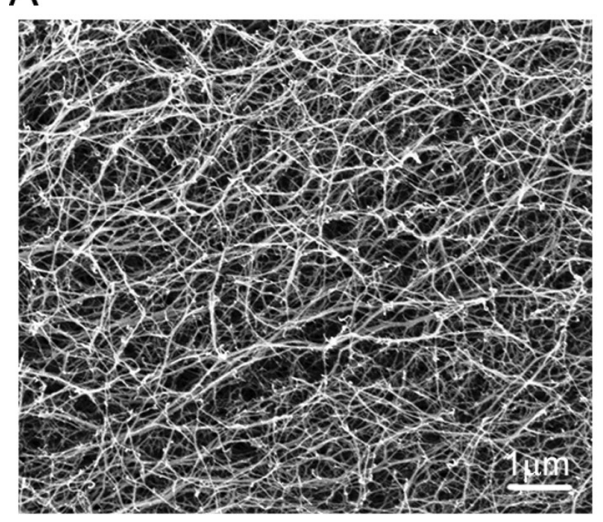

C

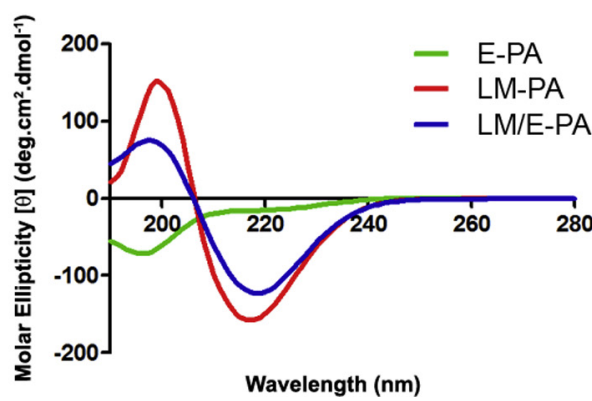

B

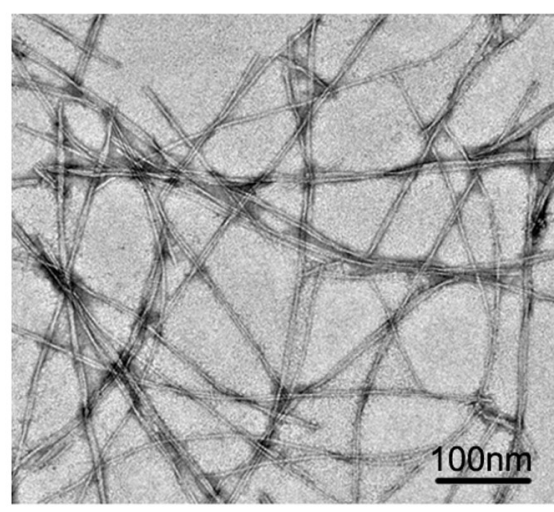

D

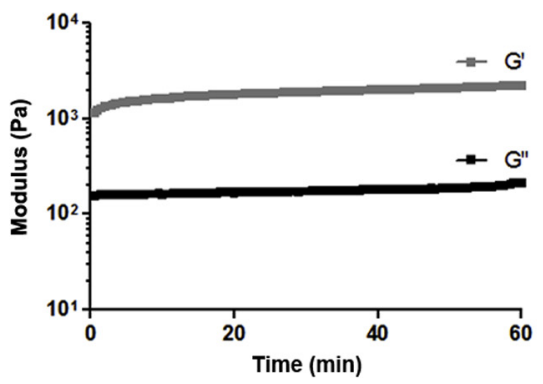

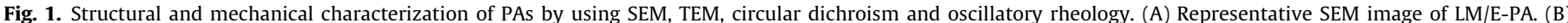

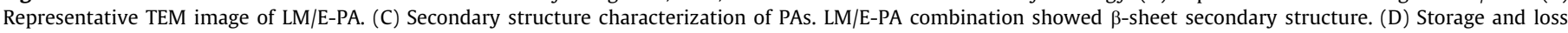
moduli of LM/E-PA. Peptides were mixed inside the syringe before taking measurements. 
A

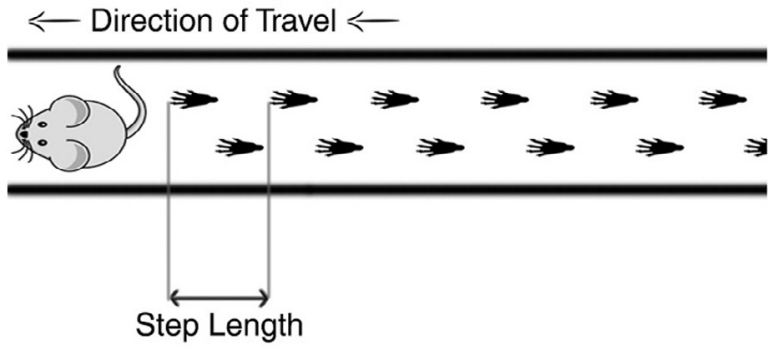

B

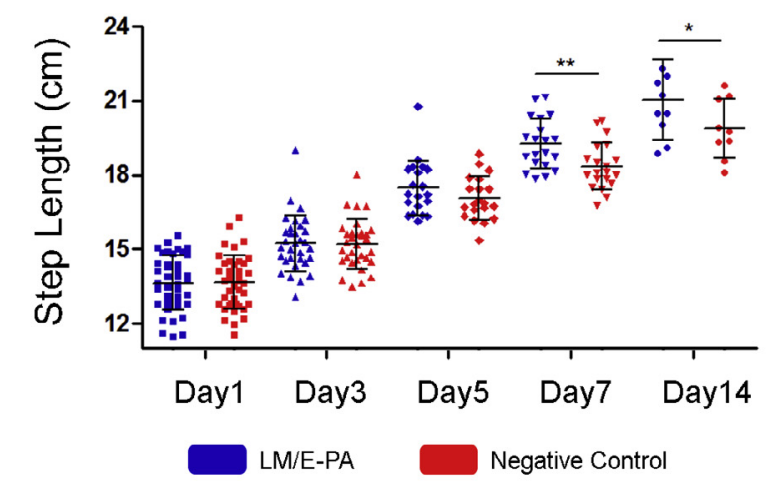

C

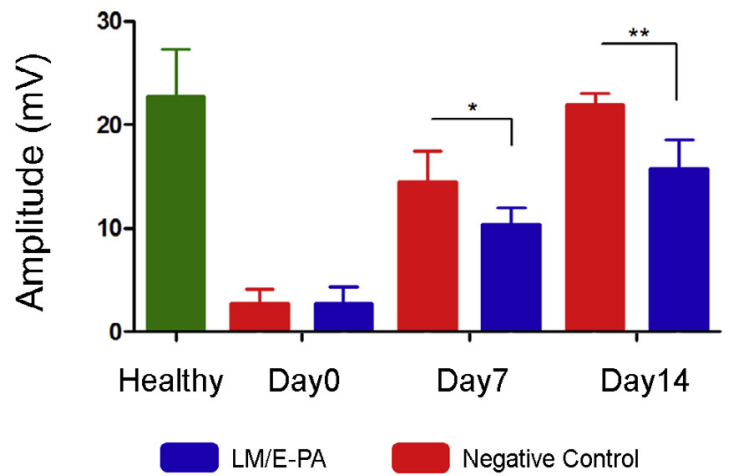

Fig. 2. Results of walking track step test and electromyography. (A) Schematic illustration of step test box and experiment setup. (B) Quantitative analysis of step length in both peptide nanofiber and negative control groups. Peptide nanofiber treated group showed increased amount of functional recovery in long term. Error bars represent mean $\pm \mathrm{SD}$. (C) Electromyography (EMG) results show PA treated group response with higher action potential in long term. Error bars represent mean $\pm \mathrm{SD}, \mathrm{n}=10\left({ }^{* * *} \mathrm{p}<0.001,{ }^{* *} \mathrm{p}<0.01,{ }^{*} \mathrm{p}<0.05\right)$.

observed at day 14 for the negative control group, further supporting the step test data. At day 14, amplitude values of peptide nanofiber treated muscle tissues were comparable to healthy muscle tissue, which suggests the significance of improvement by peptide nanofiber injection.

\subsection{Histological analyses}

We also investigated the histological appearance of muscle fibers on days 1, 3, 5, 7 and 14 after peptide nanofiber treatment (Fig. 3A). Degeneration was observed to start on day 1 and peaked on day 3 with the complete disruption of the fiber matrix. Regenerating fibers were observed on day 5 as thin fibrils, which developed into better-organized fiber bundles by day 7 . More organized fiber structure was observed in the peptide nanofiber treated group compared to the negative control group. In particular, fiber organization was complete in the bioactive nanofiber-treated group on day 14 , while fiber bundles were yet to assemble together and thicken into myofibrils in the saline-treated group on the same treatment day. Average cross section areas of muscles were also calculated from histological images (Fig. 3B). The average muscle cross section areas were similar in both peptide nanofiber and negative control groups on day 1 , while no measurements could be performed on day 3 due to the cardiotoxin-induced disruption of fibrils. However, after day 5, the bioactive peptide treated group had significantly thicker fiber cross sections compared to control group. This pattern continued for the remainder of the experimental period, and significant differences were observed between the bioactive nanofiber treated and saline groups on both day 7 and day 14 . Centrally located myonuclei images and their quantifications show that a greater number of cells merged with muscle fibers within the same period in the laminin-mimetic bioactive nanofiber applied group (Fig. 3C and D). In contrast, several satellite cells were observed not to have merged with muscle fibers in the negative control group. The percentage of myofibers with centrally located myonuclei were also significantly higher in the peptide nanofiber hydrogel treated group on day 7 . We also checked myosin heavy chain expression to evaluate the regeneration of individual muscle fibers (Supp. Fig. 5).

\subsection{Gene expression analysis}

Skeletal muscle differentiation markers were checked by qRTPCR in order to observe myogenesis at the molecular level. Four different myogenesis markers that take part in different steps of myogenesis process were used; Pax7, MyoD1, Myf5 and Myogenin. By checking these markers, the effects of peptide nanofiber on the regeneration of skeletal muscle could be further confirmed, as gene expression profiles of myogenic transcription factors are strongly related with the skeletal muscle regeneration. Primer sets that were used in this study are presented as a table in Supp. Fig. 6.

Pax7 is the main transcription factor that is expressed in satellite cells; as such, it is one of the crucial markers of early myogenesis. qRT-PCR results showed that the expression level of Pax7 gene was already high on day 1 , but reached the highest level on day 3 and its expression was increased significantly on the peptide nanofiber treated group at day 3 (Fig. 4A). However, on day 5, Pax7 expression was significantly higher in the negative control group. After day 5, Pax7 expression decreased for both groups with no significant differences between groups.

MyoD1 protein is a member of MRFs and takes a crucial part in the myogenesis process. MyoD1 expression reached its highest level on day 3 in the peptide nanofiber treated group, at which point a significant difference was present between the bioactive peptide- and saline-treated muscle tissues (Fig. 4B). This significance was also observed on day 5. On the other hand, qRT-PCR results indicated that later expression of MyoD1 was observed in the control group on day 7 and day 14 compared to peptide nanofiber treated animals.

Myf5 is another transcription factor that is responsible for the activation of satellite cells during myogenesis, and it is one of the earliest markers of myogenic differentiation. In the present study, Myf5 expression level was significantly higher in peptide nanofiber group than the negative control group on day 3 (Fig. 4C). Inversely, on day 5, Myf5 expression level was significantly lower in the peptide nanofiber group. Myf5 expression level also decreased gradually following this time point, again suggesting that myogenic gene expression occurs early in the presence of laminin mimetic peptide nanofibers and is delayed in the saline-treated group.

Myogenin is another transcription factor and is one of the latest markers of skeletal muscle regeneration. Here, high expression levels of myogenin were observed on day 7 and day 14 (Fig. 4D). 
A

Day 1

Day 3

Day 5

Day 7

Day 14

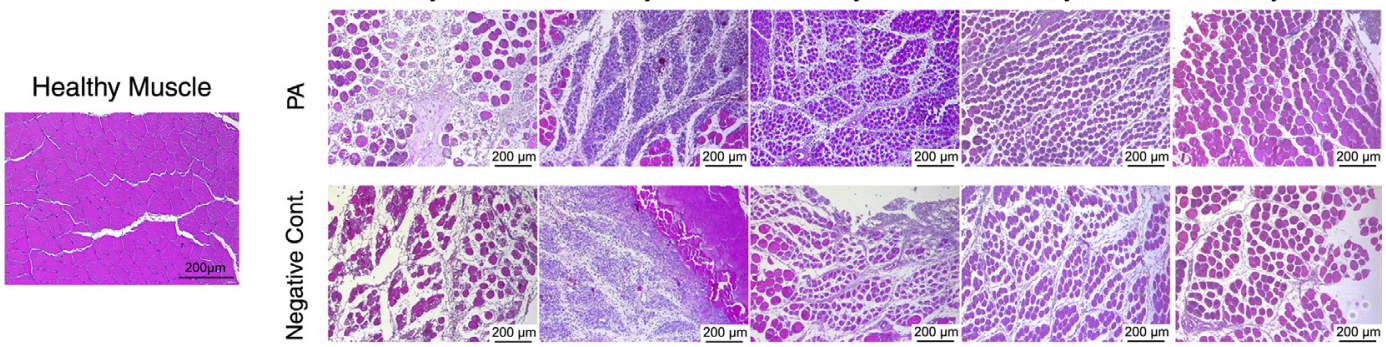

B

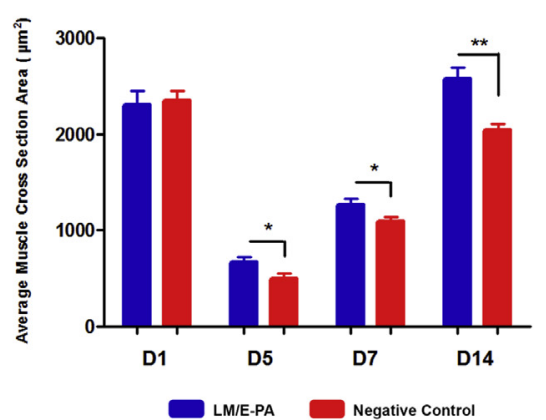

C

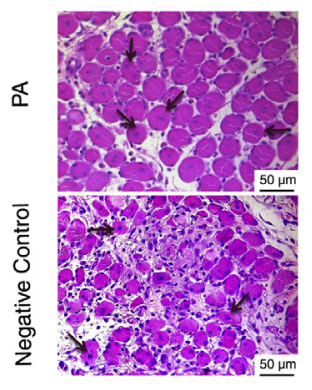

D

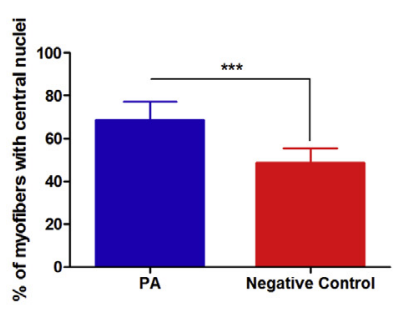

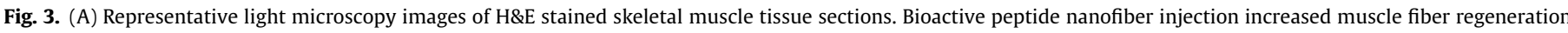

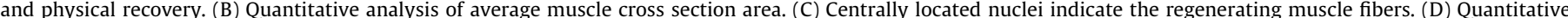

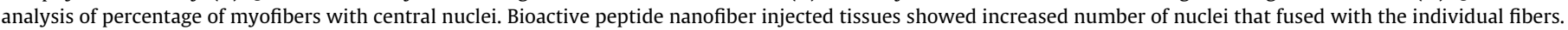
Error bars represent mean $\pm S D, n=10\left({ }^{* * *} p<0.001,{ }^{* *} p<0.01, " p<0.05\right)$.

A

$\operatorname{Pax} 7$

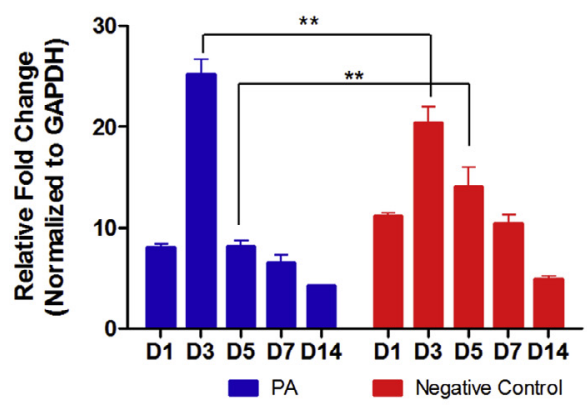

C

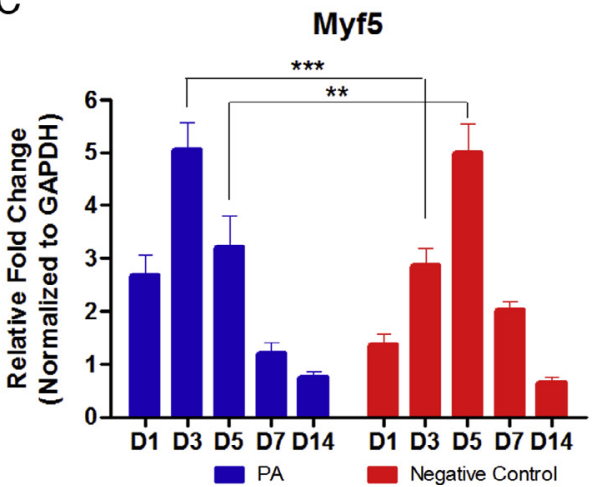

B

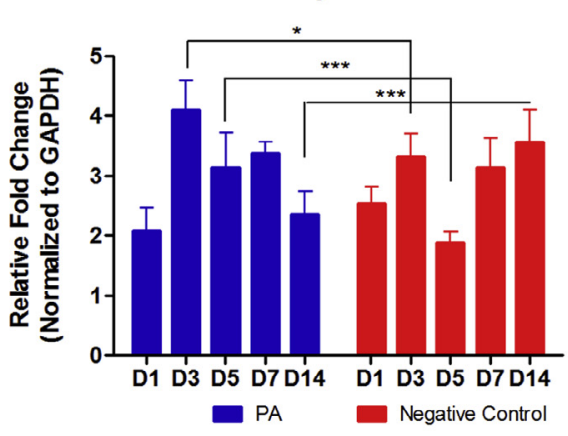

Myogenin

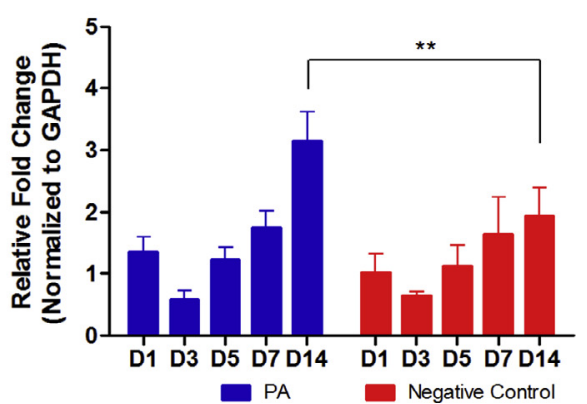

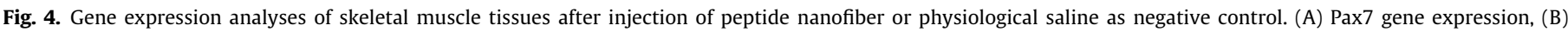

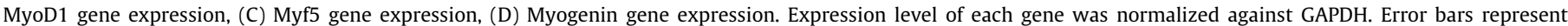
mean \pm SEM, $n=5\left({ }^{* *} \mathrm{p}<0.001,{ }^{* *} \mathrm{p}<0.01,{ }^{*} \mathrm{p}<0.05\right)$. 
In addition, day 14 expression level was significantly higher in the peptide nanofiber treated group than the negative control group. The same myogenin expression patterns were observed in both peptide nanofiber and negative control groups, but expression was generally lower in the saline-treated group on day 3 and day 5 .

\subsection{Protein detection analysis}

Western blotting was utilized to determine the expression of skeletal muscle myogenesis markers representing early, middle and late stages of myogenesis at protein level. The aim of checking these individual time points was to follow changes step by step during myogenesis and to analyze the effects of peptide nanofiber on the regeneration of skeletal muscle.

Western blotting analysis suggests that the Pax7 protein was expressed strongly on day 3 for the peptide nanofiber group (Fig. 5A). In contrast, Pax7 expression peaked on day 5 for the saline control group and decreased afterwards for both treatments, suggesting that peptide nanofiber treatment is associated with earlier expression of myogenic markers. These results are also in agreement with the qRT-PCR results.

MyoD1 shows the commitment of cell differentiation and, like Pax7, was expressed strongly in the peptide nanofiber treatment group on day 3 and in the control group on day 5 (Fig. 5B). Myf5 levels were similarly high in the nanofiber treated group on days 1 and 3 , while the negative control group exhibited a peak in Myf5 expression on day 5 (Fig. 5C).

Myogenin was also quantified as a marker of the final stages of regeneration, and the bioactive nanofiber applied group was found to exhibit significantly higher expression results when compared to the negative control group. In the present study, high expression levels of myogenin were observed after day 5 and reached their highest level on day 14, and peptide nanofiber treatment was found to increase the expression levels on days 3, 5 and 14 (Fig. 5D).

\section{Discussion}

The basal lamina of skeletal muscle plays an extremely important role in muscle regeneration. Laminin is one of the major components of skeletal muscle microenvironment, which has been previously shown to regulate the differentiation of skeletal myoblast cells [26]. Satellite cells reside in the basal lamina and are activated following injury to merge with existing fibers through the assistance of ECM proteins, which mediate the interactions between the basal lamina and skeletal muscle cells [27,28]. Mimicking ECM proteins for cellular adhesion, proliferation and differentiation is a widely used technique for regenerative medicine applications $[29,30]$. In the present study, we used the lamininderived bioactive peptide sequence IKVAV to enhance myogenesis in vivo by mimicking the basal lamina of skeletal muscle. The laminin-mimetic bioactive nanofiber system was previously shown to induce the myogenic differentiation, adhesion and growth of $\mathrm{C} 2 \mathrm{C} 12$ myoblast cells by providing a biocompatible microenvironment [16]. In addition to the IKVAV sequence, the scaffold-forming peptide amphiphile was designed to incorporate an alkyl tail and a $\beta$-sheet promoting VVAG sequence to selfassemble through hydrophobic collapse [31]. This nanofiber structure was critically designed to present a bioactive epitope at an accessible location and allow precise control over the charge of the PA molecule [32]. In the self-assembled arrangement, the hydrophobic alkyl tails comprise the core, while the hydrophilic peptide segments form a shielding outer surface, producing a cylindrical nanofiber under physiological conditions. As such, we are able to control the mechanical properties of the resulting nanofiber networks, which were designed to present a mesh-like structure similar to that found in the native ECM. TEM results showed that the lengths and thicknesses of individual PA nanofibers were uniform, and SEM images showed that the nanofiber network was morphologically similar to the natural ECM of tissues [33]. Since the strength and clemency of ECM is very important for myo-
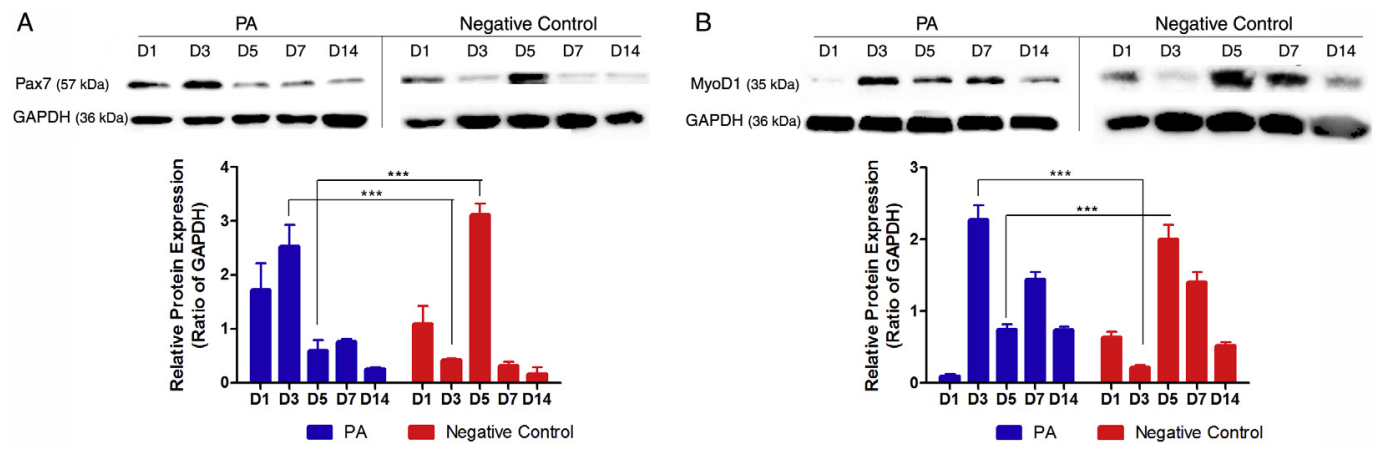

C

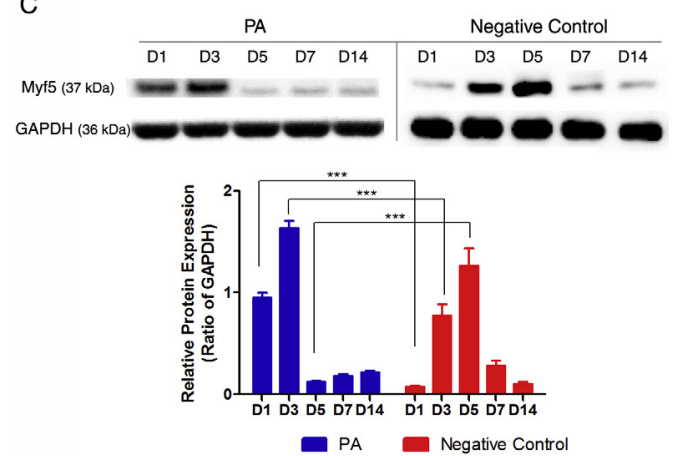

D

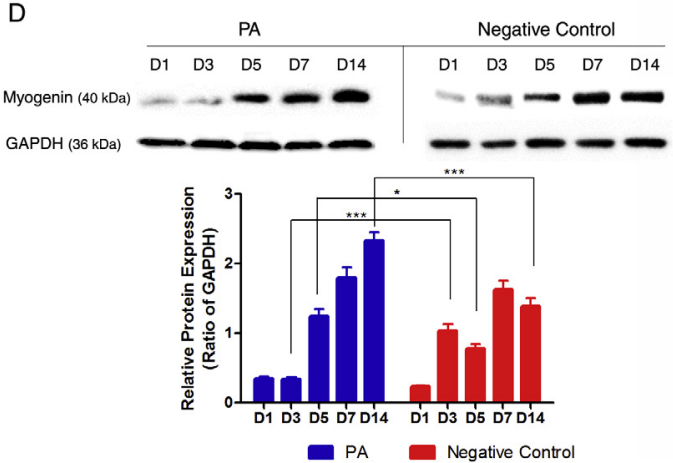

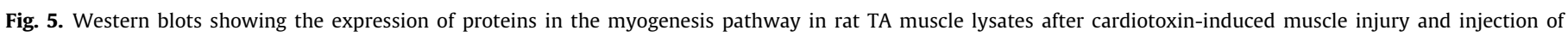

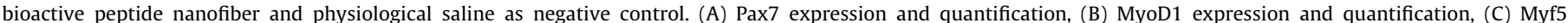

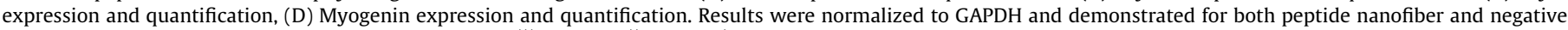
control groups. Error bars represent mean \pm SEM, $n=5\left({ }^{* * *} \mathrm{p}<0.001,{ }^{* *} \mathrm{p}<0.01,{ }^{*} \mathrm{p}<0.05\right)$. 
genic differentiation, oscillatory rheology measurements were also conducted to investigate the mechanical properties of the hydrogel, and gel formation was confirmed through this method. Storage moduli $\left(G^{\prime}\right)$ of peptide scaffolds were found to be higher than their loss moduli $\left(G^{\prime \prime}\right)$, indicating gel formation. We also checked whether mechanical properties depended on the mixing method used to prepare the hydrogels, but found no significant differences (Supp. Fig. 3).

A 14-day long experimental period was carried out to observe all overlapping phases of skeletal muscle tissue regeneration, including the formation of mature muscle fibers. We therefore did not carry out our experiments for more than 14 days to prevent unnecessary use of animals due to ethical concerns. As shorter step lengths correspond directly to the severity of muscle injury and the loss of muscle function, step tests are commonly employed to evaluate the recovery of muscle tissue in acute leg injury models. Since the tibialis anterior muscle is responsible for the dorsiflexion movement the stepping behavior of animals was directly affected by injury $[24,34]$. The behavioral step test results showed that the recovery of peptide nanofiber muscle injuries on day 7 is comparable to that of saline-treated injuries on day 14, suggesting that bioactive gel treatment greatly shortens the length of functional recovery. No significant differences were observed during the first five days of the experiment, which is attributable to the aftereffects of muscle trauma and the resulting loss in muscle mass and function.

Electromyography (EMG) is commonly used to measure the motor action potential [35]. EMG results of healthy animals were performed to evaluate the reliability of the system and were observed to be identical for both legs. Cardiotoxin (CTX) injection strongly decreased the amplitude values. As with the step length results, day 7 amplitudes of peptide nanofiber treated injuries were comparable to day 14 amplitudes of negative controls, suggesting that scaffold treatment considerably increased the rate of recovery, which suggests that laminin-mimetic peptide nanofiber treatment can be utilized to support the muscle healing process, that normally takes 3-4 weeks for completion [36,37].

Since satellite cells exhibit an exceptional capacity to renew themselves following degeneration, some amount of recovery is expected even for the saline-injected group. Indeed, muscle injury itself serves as a stimulus for the activation and proliferation of satellite cells $[3,38,39]$. However, this stimulus is often insufficient for adequate muscle repair in severe injuries, necessitating external intervention for complete recovery. LM/E-PA was therefore used as an enhancer and modulator of the inherent regenerative capacity of muscle tissue. For investigation of muscle fiber regeneration, individual skeletal muscle fibers were visualized by H\&E staining (at different time points; days 1, 3, 5, 7 and 14) and analyzed to determine their thicknesses following peptide nanofiber or saline treatment. Muscle fiber diameters were found to increase to a greater extent following peptide nanofiber treatment compared to the negative control group. Individual fibers could not be observed separately on day 3 due to post-injury degeneration of the muscle fibers [40]. After 14 days of regeneration, nearly all of the injured area was regenerated through the fusion of satellite cells with muscle fibers in both groups; however, bioactive peptide nanofiber treatment resulted in higher fiber diameters compared to the control group. Myosin heavy chain is a motor protein that generates force to drive muscle contraction. Given that it is synthesized in newly regenerated myofibers, MHC expression in the negative control was expected. Notably, the gap between fibers was greater in negative control group, indicating late myogenesis (Supp. Fig. 5).

Cross section area of skeletal muscle fibers were also calculated from histological images, with the exception of day 3 which was omitted because skeletal muscle fiber breakdown reaches a peak at this time period and interferes with the quantification method. Regeneration was found to begin by day 5 , at which point the pep- tide nanofiber group demonstrated a clear increase in fiber area compared to negative controls. Since skeletal muscle cross section area is directly related with the strength and the effective capacity of muscle [41,42], this result shows that the laminin mimetic bioactive nanofibers facilitate greater physical recovery at the histological level by accelerating the regeneration of muscle fibers.

Satellite cells are normally found in the periphery of the fiber, but their migration during muscle repair results in the presence of a considerable number of centrally located nuclei in regenerating fibers. Thus, centrally located myonuclei are a major indicator of the newly regenerated fibers $[43,44]$. In the present study, the percentage of myofibers with centrally located nuclei was significantly higher in the laminin-mimetic peptide nanofiber treated group on day 7 which suggests that bioactive nanofiber treatment accelerated skeletal muscle regeneration by facilitating satellite cell activation and fusion of these cells with the fibers (day 7 data was used because individual fibers can be observed clearly on day 7 in both PA and control groups). Our histological analyses overall suggest that laminin-mimetic PA treatment promotes the early reaction of satellite cells and facilitates enhanced skeletal muscle regeneration.

Pax7 is an early marker of skeletal myogenesis that is expressed in satellite cells under quiescence and immediately following activation $[45,46]$. Pax-7 expressing myoblast cells take an active role in the regeneration of skeletal muscle fibers, and qRT-PCR and immunoblotting results were consistent with the early activation of satellite cells in the peptide nanofiber treated group. After day 5, increased expression of other myogenic factors (MyoD1 and Myf5) leads to the decrease of Pax7 expression. Our results show that the decrease in Pax7 expression is accompanied with increased production of MyoD1, suggesting that the satellite cell activation process is well underway at day 3 onwards.

It is also known that the balance between Pax7 and Myf5 controls cell fate and the combined expression of MyoD1 and Myf5 is necessary for skeletal muscle myogenesis [47,48]. As such, the expression of Myf5 can be utilized to identify the population of muscle progenitor cells that exhibit a high tendency to differentiate. Under this metric, differentiation reached its highest level on day 3 for the peptide nanofiber treated group and on day 5 for the negative control, which further supports the idea that peptide nanofiber treatment increased the rate of regeneration at the molecular level. In addition, a transient downregulation in MyoD1 levels was observed in both LM/E-PA and control groups. This effect is attributable to the fact that MyoD1 and Myf5 perform opposing functions and exhibit a cyclic fluctuation in expression levels during muscle regeneration [48]. The downregulation of MyoD1 between two upregulations is likely to have been caused by the cyclic nature of MyoD1 and Myf5 expressions during muscle regeneration. Similarly, the increase in MyoD1 after day 7 in negative control is related to the natural ability of muscle fibers to regenerate even in the absence of bioactive peptide treatment. It is known that MyoD1 is an early marker of myogenesis, and its upregulation in the negative control shows that muscle recovery is initiated on days 5 through 14 . Similar effects were also observed for other proteins: Myf5, for example, was also upregulated on days 3 and 5 in the saline control at the gene level, while myogenin expression increased between days 3 and 14. As such, partial recovery was observed through both histological and expression analyses in the saline group, but did not occur as rapidly or strongly as in the LM/E-PA group, which showed earlier upregulation of myogenic markers and more comprehensive repair at the tissue level. The expression of myogenin, a late marker of regeneration that is found in freshly regenerated skeletal muscle fibers [49], was also strongly present on day 14 for the peptide nanofiber treated group, which suggests that skeletal muscle regeneration was at its final stages after two weeks following bioactive peptide 
nanofiber injection. Our findings overall suggest the myogenesis is supported at the molecular level by the laminin mimetic peptide nanofiber, which presumably interacts with satellite cells and facilitates their commitment to the myogenic lineage. Gene expression and protein detection analysis demonstrated that the activation of satellite cells and myogenic differentiation occurs at an early stage and is enhanced on laminin-mimetic peptide nanofibers, without any additional treatment.

\section{Conclusion}

Peptide nanofibers are biocompatible, biodegradable and nonimmunogenic, which are definitive advantages for drug applications. However, their stiffness is lower than natural tissue and they have shorter half-life. Given the relative youth of peptide-based regenerative medicine, much development still needs to take place before these hydrogels can be used in clinical application [50]. Our findings suggest that laminin-mimetic peptide nanofibers promote satellite cell activation and myogenic differentiation in vivo. Due to the physical mimicry of the ECM and multivalent presentation of laminin-mimetic chemical groups, peptide nanofiber treatment accelerated skeletal muscle regeneration by enhancing satellite cell recruitment and muscle fiber enlargement. In accordance with the literature, our results showed the early expression of Pax7, midlevel expression of Myf5/MyoD1 and late expression of Myogenin during myogenesis. In LM/E-PA treated group, this pattern was seen earlier than the negative control group and both histological and behavioral analyses supported these findings. The lamininmimetic structure was therefore observed to enhance early satellite cell differentiation by facilitating cell adhesion and providing better cell-cell communication for signal transmission events. The ECM-mimetic structure of the LM/E-PA nanofiber also provides mechanical support, creating a suitable environment for satellite cell differentiation.

Our findings suggest that the bioactive properties of the laminin mimetic bioactive scaffold are effective in facilitating the repair of acute muscle injuries. This is the first study to demonstrate the stimulation of myogenesis and muscle regeneration with laminin-mimetic bioactive peptide nanofiber scaffolds in vivo. Laminin-mimetic peptide nanofiber system does not require a linker solution to form hydrogels and instead produces nanofibers through self-assembly, which allows it to be injected directly to the site of injury. Due to its mimicry of the extracellular matrix and ease of application by injection, the laminin mimetic peptide nanofibers provide a promising material for promoting skeletal muscle regeneration for future clinical applications. However, it should be noted that another scaffold-based material would serve as a more adequate control than physiological saline to demonstrate the efficiency of the LM/E-PA system under in vivo conditions, and while a non-bioactive PA scaffold (K/E-PA) was previously shown to be ineffective in inducing myoblast differentiation in vitro, another laminin-based scaffold may be evaluated for this purpose in further studies.

\section{Author contributions}

C.E.C., G.U., M.O.G. and A.B.T. designed the study. C.E.C., G.U. and O.U. performed research. E.K.U. conducted EMG analysis. C.E.C, O. U. and F.Y. carried out animal experiments and histology analyses. C.E.C., O.U. and E.K.U. analyzed data. M.O.G. and A.B.T. supervised research. C.E.C., M.O.G. and A.B.T. wrote the paper.

\section{Conflict of interest}

The authors declare no conflict of interest.

\section{Acknowledgements}

We would like to thank M. Guler for TEM imaging and Z. Erdogan for LC-MS analysis of peptide amphiphiles. We also would like to thank Dr. Ece Unlu for advisory on EMG analyses, Ibrahim Ulusoy for his valuable help in animal care, Alper D. Ozkan for critical discussion, Gokhan Gunay for preparing abstract figure and M. Ezgi Kaya for contributions on histology analyses. Cagla Eren Cimenci is supported by TUBITAK BIDEB 2210-C graduate fellowship.

\section{Appendix A. Supplementary data}

Supplementary data associated with this article can be found, in the online version, at http://dx.doi.org/10.1016/j.actbio.2017.07. 010.

\section{References}

[1] W.M. Han, Y.C. Jang, A.J. García, Engineered matrices for skeletal muscle satellite cell engraftment and function, Matrix Biol. (2016).

[2] Z. Yablonka-Reuveni, The skeletal muscle satellite cell: still young and fascinating at 50, J. Histochem. Cytochem. 59 (12) (2011) 1041-1059.

[3] N.A. Dumont, Y.X. Wang, M.A. Rudnicki, Intrinsic and extrinsic mechanisms regulating satellite cell function, Development 142 (9) (2015) 1572-1581.

[4] K. Tsumagari, S.C. Chang, M. Lacey, C. Baribault, S.V. Chittur, J. Sowden, R. Tawil, G.E. Crawford, M. Ehrlich, Gene expression during normal and FSHD myogenesis, BMC Med. Genomics 4 (2011) 67.

[5] H. Yin, F. Price, M.A. Rudnicki, Satellite cells and the muscle stem cell niche, Physiol. Rev. 93 (1) (2013) 23-67.

[6] T.A. Järvinen, T.L. Järvinen, M. Kääriäinen, H. Kalimo, M. Järvinen, Muscle injuries: biology and treatment, Am. J. Sports Med. 33 (5) (2005) 745-764.

[7] M. Juhas, N. Bursac, Engineering skeletal muscle repair, Curr. Opin. Biotechnol. 24 (5) (2013) 880-886

[8] L. Ricotti, A. Polini, G.G. Genchi, G. Ciofani, D. Iandolo, H. Vazão, V. Mattoli, L. Ferreira, A. Menciassi, D. Pisignano, Proliferation and skeletal myotube formation capability of $\mathrm{C} 2 \mathrm{C} 12$ and $\mathrm{H} 9 \mathrm{c} 2$ cells on isotropic and anisotropic electrospun nanofibrous PHB scaffolds, Biomed. Mater. 7 (3) (2012) 035010.

[9] C. Fuoco, L.L. Petrilli, S. Cannata, C. Gargioli, Matrix scaffolding for stem cell guidance toward skeletal muscle tissue engineering, J. Orthop. Surg. Res. 11 (1) (2016) 86.

[10] H. Geckil, F. Xu, X. Zhang, S. Moon, U. Demirci, Engineering hydrogels as extracellular matrix mimics, Nanomedicine (London) 5 (3) (2010) 469-484.

[11] S. Thorsteinsdóttir, M. Deries, A.S. Cachaço, F. Bajanca, The extracellular matrix dimension of skeletal muscle development, Dev. Biol. 354 (2) (2011) 191-207.

[12] K.I. Gawlik, M. Durbeej, Skeletal muscle laminin and MDC1A: pathogenesis and treatment strategies, Skeletal Muscle 1 (1) (2011) 9.

[13] J.R. Sanes, The basement membrane/basal lamina of skeletal muscle, J. Biol. Chem. 278 (15) (2003) 12601-12604.

[14] Y. Yao, E.H. Norris, C.E. Mason, S. Strickland, Laminin regulates PDGFR $\beta(+)$ cell stemness and muscle development, Nat. Commun. 7 (2016) 11415.

[15] C.F. Bentzinger, Y.X. Wang, J. von Maltzahn, V.D. Soleimani, H. Yin, M.A. Rudnicki, Fibronectin regulates Wnt7a signaling and satellite cell expansion, Cell Stem Cell 12 (1) (2013) 75-87.

[16] I.C. Yasa, N. Gunduz, M. Kilinc, M.O. Guler, A.B. Tekinay, Basal lamina mimetic nanofibrous peptide networks for skeletal myogenesis, Sci. Rep. 5 (2015) 16460.

[17] K. Tashiro, G.C. Sephel, B. Weeks, M. Sasaki, G.R. Martin, H.K. Kleinman, Y. Yamada, A synthetic peptide containing the IKVAV sequence from the A chain of laminin mediates cell attachment, migration, and neurite outgrowth, J. Biol. Chem. 264 (27) (1989) 16174-16182.

[18] J.D. Hartgerink, E. Beniash, S.I. Stupp, Peptide-amphiphile nanofibers: a versatile scaffold for the preparation of self-assembling materials, Proc. Natl. Acad. Sci. U.S.A. 99 (8) (2002) 5133-5138.

[19] M.J. Webber, J.A. Kessler, S.I. Stupp, Emerging peptide nanomedicine to regenerate tissues and organs, J. Intern. Med. 267 (1) (2010) 71-88.

[20] C.J. Newcomb, S. Sur, J.H. Ortony, O.S. Lee, J.B. Matson, J. Boekhoven, J.M. Yu, G. C. Schatz, S.I. Stupp, Cell death versus cell survival instructed by supramolecular cohesion of nanostructures, Nat. Commun. 5 (2014) 3321.

[21] R. Mammadov, B. Mammadov, S. Toksoz, B. Aydin, R. Yagci, A.B. Tekinay, M.O Guler, Heparin mimetic peptide nanofibers promote angiogenesis, Biomacromolecules 12 (10) (2011) 3508-3519.

[22] E. Arslan, I.C. Garip, G. Gulseren, A.B. Tekinay, M.O. Guler, Bioactive supramolecular peptide nanofibers for regenerative medicine, Adv. Healthc. Mater. 3 (9) (2014) 1357-1376.

[23] R.B. Merrifield, Solid-phase peptide syntheses, Endeavour 24 (1965) 3-7.

[24] P.O. Fernagut, E. Diguet, B. Labattu, F. Tison, A simple method to measure stride length as an index of nigrostriatal dysfunction in mice, J. Neurosci. Methods 113 (2) (2002) 123-130. 
[25] S.E. Paramonov, H.W. Jun, J.D. Hartgerink, Self-assembly of peptideamphiphile nanofibers: the roles of hydrogen bonding and amphiphilic packing, J. Am. Chem. Soc. 128 (22) (2006) 7291-7298.

[26] U. Kühl, M. Ocalan, R. Timpl, K. von der Mark, Role of laminin and fibronectin in selecting myogenic versus fibrogenic cells from skeletal muscle cells in vitro, Dev. Biol. 117 (2) (1986) 628-635.

[27] N. Osses, E. Brandan, ECM is required for skeletal muscle differentiation independently of muscle regulatory factor expression, Am. J. Physiol. Cell Physiol. 282 (2) (2002) C383-C394.

[28] S. Joanisse, J.P. Nederveen, T. Snijders, B.R. McKay, G. Parise, Skeletal muscle regeneration repair and remodelling in aging: the importance of muscle stem cells and vascularization, Gerontology 63 (1) (2017) 91-100.

[29] S. Kocabey, H. Ceylan, A.B. Tekinay, M.O. Guler, Glycosaminoglycan mimetic peptide nanofibers promote mineralization by osteogenic cells, Acta Biomater. 9 (11) (2013) 9075-9085.

[30] A. Skardal, M. Devarasetty, H.W. Kang, I. Mead, C. Bishop, T. Shupe, S.J. Lee, J. Jackson, J. Yoo, S. Soker, A. Atala, A hydrogel bioink toolkit for mimicking native tissue biochemical and mechanical properties in bioprinted tissue constructs, Acta Biomater. 25 (2015) 24-34.

[31] H. Cui, M.J. Webber, S.I. Stupp, Self-assembly of peptide amphiphiles: from molecules to nanostructures to biomaterials, Biopolymers 94 (1) (2010) 1-18.

[32] H. Ceylan, A.B. Tekinay, M.O. Guler, Selective adhesion and growth of vascular endothelial cells on bioactive peptide nanofiber functionalized stainless steel surface, Biomaterials 32 (34) (2011) 8797-8805.

[33] A.R. Gillies, R.L. Lieber, Structure and function of the skeletal muscle extracellular matrix, Muscle Nerve 44 (3) (2011) 318-331.

[34] R. Souron, A. Farabet, L. Féasson, A. Belli, G.Y. Millet, T. Lapole, Effects of an 8week local vibration training on corticospinal properties of the tibialis anterior muscle, Ann. Phys. Rehabil. Med. 59S (2016) e52.

[35] A.C. Nepomuceno, E.L. Politani, E.G. Silva, R. Salomone, M.V. Longo, A.G. Salles, J.C. Faria, R. Gemperli, Tibial and fibular nerves evaluation using intraoperative electromyography in rats, Acta Cir. Bras. 31 (8) (2016) 542-548.

[36] J. Ilha, R.T. Araujo, T. Malysz, E.E. Hermel, P. Rigon, L.L. Xavier, M. Achaval, Endurance and resistance exercise training programs elicit specific effects on sciatic nerve regeneration after experimental traumatic lesion in rats, Neurorehabil. Neural Repair 22 (4) (2008) 355-366.
[37] T.A. Järvinen, M. Järvinen, H. Kalimo, Regeneration of injured skeletal muscle after the injury, Muscles Ligaments Tendons J. 3 (4) (2013) 337-345.

[38] A.S. Brack, T.A. Rando, Tissue-specific stem cells: lessons from the skeletal muscle satellite cell, Cell Stem Cell 10 (5) (2012) 504-514.

[39] J. Scharner, P.S. Zammit, The muscle satellite cell at 50: the formative years, Skeletal Muscle 1 (1) (2011) 28.

[40] S. Ostrovidov, X. Shi, R.B. Sadeghian, S. Salehi, T. Fujie, H. Bae, M. Ramalingam, A. Khademhosseini, Stem cell differentiation toward the myogenic lineage for muscle tissue regeneration: a focus on muscular dystrophy, Stem Cell Rev. 11 (6) (2015) 866-884.

[41] P. Schantz, E. Randall-Fox, W. Hutchison, A. Tydén, P.O. Astrand, Muscle fibre type distribution, muscle cross-sectional area and maximal voluntary strength in humans, Acta Physiol. Scand. 117 (2) (1983) 219-226.

[42] F. Liu, C.S. Fry, J. Mula, J.R. Jackson, J.D. Lee, C.A. Peterson, L. Yang, Automated fiber-type-specific cross-sectional area assessment and myonuclei counting in skeletal muscle, J. Appl. Physiol. (1985) 115 (11) (2013) 1714-1724.

[43] E.S. Folker, M.K. Baylies, Nuclear positioning in muscle development and disease, Front. Physiol. 4 (2013) 363.

[44] A.L. Mazzotti, D. Coletti, The need for a consensus on the locution "Central Nuclei” in striated muscle myopathies, Front. Physiol. 7 (2016) 577.

[45] J. Reimann, K. Brimah, R. Schröder, A. Wernig, J.R. Beauchamp, T.A. Partridge, Pax7 distribution in human skeletal muscle biopsies and myogenic tissue cultures, Cell Tissue Res. 315 (2) (2004) 233-242.

[46] P. Seale, L.A. Sabourin, A. Girgis-Gabardo, A. Mansouri, P. Gruss, M.A. Rudnicki, Pax7 is required for the specification of myogenic satellite cells, Cell 102 (6) (2000) 777-786.

[47] S. Kuang, K. Kuroda, F. Le Grand, M.A. Rudnicki, Asymmetric self-renewal and commitment of satellite stem cells in muscle, Cell 129 (5) (2007) 999-1010.

[48] M.A. Rudnicki, P.N. Schnegelsberg, R.H. Stead, T. Braun, H.H. Arnold, R Jaenisch, MyoD or Myf-5 is required for the formation of skeletal muscle, Cell 75 (7) (1993) 1351-1359.

[49] Z. Yan, S. Choi, X. Liu, M. Zhang, J.J. Schageman, S.Y. Lee, R. Hart, L. Lin, F.A. Thurmond, R.S. Williams, Highly coordinated gene regulation in mouse skeletal muscle regeneration, J. Biol. Chem. 278 (10) (2003) 8826-8836.

[50] P. Worthington, D.J. Pochan, S.A. Langhans, Peptide hydrogels - versatile matrices for 3D cell culture in cancer medicine, Front. Oncol. 5 (2015) 92. 